\title{
Percepción del posicionamiento estratégico en el ámbito de las franquicias de comida rápida en España
}

\author{
Schlesinger Díaz, María Walesska*
}

\section{Resumen}

La tipología de posicionamiento estratégico de Miles y Snow constituye una herramienta útil para categorizar las empresas en base al tipo de estrategias que siguen. La escala desarrollada por Aragon (1996) para categorizar las empresas a partir de la propuesta de Miles y Snow (1978) se aplicó al contexto de servicios específicamente de comida rápida, a través de un muestreo sistemático y seleccionando una muestra representativa de los gerentes de tienda de las franquicias de comida rápida de la Comunidad Valenciana en España. Luego de la categorización de las 37 unidades muestrales compuestas por las franquicias correspondientes a 7 tipos de restaurantes de comida rápida y a través de un análisis ANOVA de las medias se contrastaron las hipótesis según las cuales determinadas categorías tienen más tendencia a desarrollar capacidades de marketing y de innovación. Se concluye que en las tres tipologías detectadas los gerentes no perciben diferencias significativas en relación a la innovación, pero por otra parte si se encontraron diferencias significativas en relación al énfasis que dan al marketing las empresas analizadoras y prospectoras en comparación con las defensivas. Los gerentes deben evaluar la consistencia entre la tipología a la cual pertenecen y los elementos de la estrategia empleada.

Palabras clave: Tipologías estratégicas, marketing, innovación, Miles y Snow, posicionamiento.

\section{Perception of Strategic Positioning in Fast Food Services, Spain}

\begin{abstract}
The Miles and Snow (1978) strategic positioning typology is a useful tool for categorizing companies based on the types of strategies they follow. The scale developed by Aragon (1996) for categorizing companies based on Miles and Snow's (1978) proposal was applied to the context of services, specifically for fast food, using systematic sampling and selecting a representative sam-

Recibido: 18-01-07. Aceptado: 18-01-08

* Docente-Investigadora de la Facultad de Ciencias Económicas y Sociales de la Universidad del Zulia. E-mail: walesskas@cantv.net
\end{abstract}


ple of franchise fast food store managers in the community of Valencia, Spain. After categorizing the 37 sample units composed of franchises corresponding to 7 fast food restaurant types and using an ANOVA analysis of the means, hypotheses were contrasted according to which certain categories had a greater tendency to develop marketing and innovation capacities. Conclusions were that for the three typologies detected, managers do not perceive significant differences related to innovation, but on the other hand, significant differences were found related to the emphasis that analytical and prospecting companies give to marketing when compared to defensive companies. Managers should evaluate the consistency between the typology to which their company belongs and the strategy elements used.

Key words: Strategic typologies, marketing, innovation, Miles and Snow, positioning.

\section{Introducción}

El término posicionamiento es un término subjetivo y relativo, puesto que se ha definido a lo largo del tiempo en líneas generales como el lugar que ocupa un producto o servicio en la mente del consumidor y que lo diferencia de sus competidores. Trout habló por primera vez de posicionamiento en 1969 y desde entonces junto a Al Ries se consideran los padres de este término, refiriéndose a él como todo lo que se hace con la mente de los probables clientes; o sea, como se ubica el producto en la mente de éstos. Según Trout (1969) una empresa puede abrirse paso en un sector, incluso en el mercado internacional, pero su esfuerzo siempre será en vano si su imagen propia (de marca, que dirían algunos) no consigue crearse un hueco en la mente del consumidor. Este concepto es aplicable a cualquier actividad en la que medie un entorno competitivo (Trout, 1969). De esta manera cada empresa particularmente establece estrategias que buscan posicionarla de alguna forma específica en el mercado, y es un punto clave para ellas, puesto que puede influir en su desempeño dentro de la industria a la cual pertenece.
A partir del surgimiento de este término, numerosos autores han planteado diversos enfoques sobre como se puede posicionar un producto, marca o empresa. Entre los más destacados se encuentran Porter (1985), Ries y Jack Trout (1981), Walker y Ruekert (1987) Chrisman, Hofer y Boulton (1988) y Miles y Snow (1978), todos ellos han propuesto diversas clasificaciones sobre el posicionamiento estratégico algunos en mayor o menor medida tienen vigencia actualmente.

En el presente trabajo se ha seleccionado la tipología de Miles y Snow (1978), la cual surge del análisis de las relaciones de la empresa con su entorno y las relaciones entre la estrategia y su estructura.

Asimismo se presenta un estudio empírico enfocado en el posicionamiento de una organización de acuerdo a la estrategia de nivel de negocio de una empresa, aplicando la tipología propuesta por Miles y Snow, al sector de las franquicias de comida rápida en España, específicamente en la Comunidad Valenciana, con la finalidad última de conectar cada tipología con la estrategia del negocio en base a la innovación y capacidades del marketing de las empresas de este sector. 
Posicionamiento estratégico en el ámbito de las franquicias de comida rápida Schlesinger Díaz, María Walesska

La tipología de Miles y Snow (1978) ha sido objeto de numerosas investigaciones y ha tenido gran incidencia en la literatura estratégica al haber sido aplicada directamente a la explicación del comportamiento de un gran número de organizaciones de diferentes sectores (Mc. Daniel y Kolari, 1987, Morgan, Strong y Mc. Guiness, 2001, Hambrick, 1982 y Pearce y Zahra, 1990).

Asimismo, ha servido de base a numerosos estudios en el campo de la dirección estratégica ya que incluye, en su definición, dimensiones de estrategia, estructura y proceso, todas ellas de gran interés en el citado campo. Si bien este modelo de tipologías surge con muy poca evidencia empírica, tal y como reconocen sus autores, posteriormente se ha realizado una amplia investigación que le ha dado un gran soporte, justificando su utilidad.

Hambrick (1980 y 1982) utiliza esta tipología por su base empírica en diferentes industrias, por permitir la identificación de estrategias extremas en distintas industrias y por la riqueza de sus hipótesis teóricas sobre distintas relaciones de interés. En el mismo sentido muchos autores describen a la tipología de Miles y Snow (1978) como bien aceptada académicamente e internamente consistente (Conant, Mokwan y Varadarajan 1990; Segev 1989; Shortell y Zajac, 1990). Según Mc Daniel y Kolari (1987) este enfoque contempla la organización como un sistema completo e integrado, en interacción dinámica con el entorno. Por otro lado Shortell y Zajac, 1990; Nutt y Bachoff, 1992) enfatizan la capacidad de encapsular la estrategia a un nivel de abstracción suficiente para aplicarla a una gran variedad de organizaciones y sectores.
Por su doble utilidad organizativa y estratégica, la tipología de Miles y Snow ha sido repetidamente utilizada y consensuada y, a pesar de que la tipología de Porter es más apropiada para industrias concentradas y unidades de negocio grandes y unidades de negocio grandes con más competidores, algunos estudios establecen un paralelismo entre la tipología de Miles y Snow y la tipología de Porter, asociando la diferenciación de productos con la estrategia exploradora y la estrategia de bajos costes con la estrategia del defensor (García y Pérez, 2001).

Además de estas razones, su amplia utilización en la literatura sobre estrategia y el hecho que las tipologías sean claras y relativamente sencillas de identificar son los motivos por los cuales se ha seleccionado la Tipología de Miles y Snow para la siguiente investigación.

El presente trabajo pretende abordar como objetivos la caracterización de las empresas de franquicias de comida rápida españolas de acuerdo a la clasificación tipológica de Miles y Snow (1978) sobre orientación/posición estratégica y comparar la capacidad de innovación y de marketing en cada una de las tipologías planteadas por Miles y Snow: prospectoras, analizadoras, defensivas $y$ reactivas.

\section{Planteamientos Teóricos}

En este apartado se presenta, de forma sucinta, la revisión de la literatura y se plantean las hipótesis de trabajo; en primera instancia se aborda la parte correspondiente al posicionamiento estratégico y luego específicamente la tipología seleccionada para el estudio. 


\subsection{Posicionamiento-Estrategia}

Según Ries y Trout (2000) hay una palabra que los empresarios no pueden olvidar: No pueden ser ambiguos, es decir, no pueden abarcar muchos aspectos, los consumidores deben identificar sus productos con algo en especial, para lograr un claro posicionamiento en su mente. Ciertos autores (Ramsay, 1983; Mc Alexander, Becker y Kaldenberg, 1993) confirman la importancia del posicionamiento por la relación que existe entre el éxito y crecimiento que la empresa pueda obtener y el estar bien posicionado en el mercado.

Ahora bien, para lograr este posicionamiento las empresas se deben basar en una estrategia claramente definida. Las definiciones de estrategia son innumerables e incluso con diferencias de alcance, diversos autores coinciden en que la estrategia es la que guía la toma de decisiones y las relaciones entre la empresa y su entorno, afecta la estructura y los procesos dentro de la organización y como consecuencia los resultados que pueda obtener en su actividad (Andrews 1977, Argyris 1985, Mintzberg 1978 y Porter 1980).

A nivel empresarial, la estrategia es sinónimo de orientación estratégica que comúnmente es definida como: la forma en la cual una organización usa la estrategia para adaptar o cambiar aspectos de su entorno a su favor. (Manu y Sriram, 1996).

En una situación como la actual, caracterizada por la globalización y la internacionalización de los mercados, es fundamental para las empresas conseguir una posición competitiva fuerte sobre sus rivales, para ello es necesario elegire implantar la estrategia con más probabilidades de éxito. La etapa de la formulación de la estrategia recoge la elección de una estrategia, esta elección no se puede hacer al azar o por intuición, para realizarla, es necesario tener un conjunto de posibles estrategias entre las cuales seleccionar una. Existe un abanico muy amplio de posibilidades para adquirir esa posición privilegiada, es decir, existen diferentes tipos de estrategias, ello se debe, fundamentalmente, a la gran cantidad de clasificaciones o tipologías de estrategias propuestas en la literatura, casi tantas como investigadores centrados en el campo de la estrategia empresarial (Miles y Snow, 1978; Abell, 1980; Mintzberg, 1988; Galbraith y Schendel, 1983).

Existen diversas clasificaciones algunas de ellas se centran en el estudio de la estrategia a nivel de unidad de negocio, otros además de la unidad de negocio también estudian los distintos tipos de estrategias a nivel corporativo y otras clasificaciones, además de las dos anteriores tipologías, también recogen las formas de llevar a cabo estos dos niveles de estrategia (García y Sabater, 2003).

Según García y Sabater (2003) la estrategia corporativa es aquella que contempla a la empresa como un todo, aunque la empresa tenga distintos negocios en distintos sectores y la estrategia de negocio es aquella específica de cada una de las actividades llevadas a cabo por la empresa, sería el plan de actuación para cada negocio. En la estrategia a nivel de unidad de negocio, lo esencial es la construcción y sostenimiento de una ventaja competitiva, el fortalecimiento de esa ventaja competitiva a largo plazo será su objetivo esencial. 
Posicionamiento estratégico en el ámbito de las franquicias de comida rápida Schlesinger Díaz, María Walesska

Centrándonos en las clasificaciones que recoge García y Sabater (2003), entre los autores que centran sus estudios en la estrategia a nivel de unidad de negocio, la selección de estas clasificaciones ha estado en función de la repercusión de las mismas en trabajos de investigación posteriores, Miles y Snow (1978), Abell (1980), Porter (1985) y Beal (2000), pero también hay clasificaciones que aunque están centradas a nivel corporativo definen algún tipo de estrategia a nivel de unidad de negocio, autores como: Galbraith y Schendel (1983) o Mintzberg (1988). Estos enfoques se recogen en el Cuadro 1.

Como se puede notar, los tipos de estrategia que más se repiten o utilizan son: una estrategia volcada en la reducción de costes, una estrategia basada en ser diferente a la competencia y un tipo de estrategia centrado en un segmento, cuya ventaja competitiva es: reducir costes, diferenciarme o ambas.

La estrategia de liderazgo en costes se correspondería con la "Defensora" que plantean Miles y Snow (1978). Se trata de una estrategia que se caracteriza por tener tecnología intensiva en capital y eficiencia en la producción, ingeniería de procesos y control de costes. En la clasificación de Abell (1980) no se encuentra ningún tipo que se asemeje a la de liderazgo en costes. Porter (1982), define claramente una estrategia de liderazgo en costes. En este sentido, Galbraith y Schendel (1983) definen la estrategia "Mantenimiento" cuya finalidad consiste también en la reducción de costes.

Otro tipo de estrategia definido, es la estrategia de Diferenciación, caracterizada por ser una estrategia centrada en conseguir distinguir el producto o servicio que ofrece la empresa respecto a la competencia. Las formas de diferenciar pueden estar basadas en la innovación, ventas, calidad o servicio (Beal, 2000). La estrategia Prospectiva de Miles y Snow (1978), es una estrategia que está buscando continuamente nuevas oportunidades, nuevos productos o mercados. Por eso podríamos identificarla con la estrategia de diferenciación definida anteriormente. Así mismo, la clasificación de Abell (1980) sí contempla la estrategia de "Diferenciación" con características simi-

\section{Cuadro 1 Tipos de estrategia}

\begin{tabular}{ll}
\hline Tipos de estrategia & Autores \\
\hline Liderazgo en Costes & Miles y Snow (1978); Porter (1982); \\
& Galbraith Schendel (1983) \\
Diferenciación & Miles y Snow (1978); Porter (1982); \\
& Mintzberg (1988); Beal (2000); \\
Enfoque & Miles y Snow (1978); Porter (1982); Galbraith y Schendel \\
& (1983); Mintzberg (1988) \\
Analizadora & Miles y Snow (1978) \\
No Diferenciación & Abell (1980); Galbraith Schendel (1983) \\
\hline
\end{tabular}

Fuente: Elaboración propia a partir de García y Sabater (2003). 
lares a las descritas. Porter (1982) también lo hace. Adicionalmente, la estrategia de "Diferenciación", definida por Mintzberg (1988) define una única estrategia, la de diferenciación asimila el bajo coste a: costes bajos en imagen, servicio, calidad o diseño.

La tercera estrategia es la de Enfoque, definida como aquella estrategia cuyo objetivo es centrarse en un grupo de compradores en particular, en un segmento de la línea del producto, o en un mercado geográfico determinado. No existe una alternativa dentro de la clasificación de Miles y Show (1978) con estas características. Sin embargo, para Abell (1980), existe una estrategia clara de "Enfoque". En la misma línea, Porter (1982) define una estrategia de este tipo especificando que la ventaja competitiva que resalta la empresa, una vez segmentado el mercado es: costes o diferenciación. Así mismo, Galbraith y Schendel (1983), definen tanto para empresas de consumo como industriales, una estrategia de "Nicho", siendo una estrategia de especialización en calidad, o de enfoque por diferenciación. Mintzberg (1988) también, define la estrategia de "Alcance", pero al igual que la estrategia de diferenciación, su tipo de estrategia es más detallada que la de "Enfoque" definida anteriormente.

Además de los tres tipos de estrategias vistas anteriormente, liderazgo en costes, diferenciación y enfoque, diversos autores añaden estrategias propias. Así, la clasificación de Miles y Show (1978) añade la estrategia "analizadora" cuyas características están situadas entre la defensora y prospectiva, y su rasgo principal es imitar lo que hace el resto de la competencia. Abell (1980) agrega la estrategia de "No-Diferenciación", contraria a la estrategia de "enfoque" caracterizada por un amplio alcance pero donde no tiene una segmentación diferenciada. Por su parte Galbraith y Schendel (1983) incorporan la estrategia "Escaladora" aplicada por empresas que se caracterizan por tener bajos precios, baja calidad y poca línea de productos, asemejándose a una estrategia de no-diferenciación. Dentro del apartado de empresas industriales, está la estrategia de "Bajo Compromiso" que es semejante a la estrategia escaladora definida anteriormente.

Una vez que se ha planteado claramente las diferentes alternativas, que tienen las empresas de posicionarse en el mercado, se desarrollará más específicamente el modelo de posicionamiento estratégico planteado por Miles y Snow (1978) por permitir la identificación de estrategias extremas en distintas industrias y por la riqueza de sus hipótesis teóricas sobre distintas relaciones de interés y haber sido objeto de diversos estudios en diferentes tipos de industrias.

\subsection{Tipologías Estratégicas: Miles y Snow (1978)}

La tipología de estrategias de negocios de Miles y Snow (1978) está basada en tres grandes hipótesis:

La primera de ellas es que las organizaciones con éxito desarrollan a lo largo del tiempo una adaptación sistemática al entorno. Este ciclo de adaptación refleja las distintas aproximaciones de las organizaciones para enfrentarse a tres tipos de problemas en su entorno competitivo: 
Posicionamiento estratégico en el ámbito de las franquicias de comida rápida Schlesinger Díaz, María Walesska

problemas de empresa, relacionados con la definición de las combinaciones producto-mercado en las que operan; problemas de ingeniería, centrados en el sistema técnico de la organización y problemas administrativos, relativos a los asuntos de estructura y procesos.

1. Problema Emprendedor

Su solución pasa por la aceptación, por parte de la dirección, de un dominio concreto producto-mercado, y esa aceptación resulta evidente cuando la dirección decide comprometer recursos para alcanzar los objetivos relativos al dominio aceptado. La función emprendedora es responsabilidad de la alta dirección, y debe comprometer tiempo y otros recursos.

\section{Problema de Ingeniería}

Implica la creación, con la dirección actual de operaciones, de un sistema que dé solución al problema emprendedor. La creación de este sistema exige que la dirección decida la tecnología apropiada para producir y distribuir los productos / servicios escogidos y formar los nuevos vínculos de información, comunicación y control que aseguren el uso adecuado de la tecnología.

\section{Problema Administrativo}

Consiste en racionalizar y estabilizar aquellas actividades que resuelven bien los problemas abordados por la organización en las fases emprendedora y de ingeniería. Solucionar el problema administrativo es algo más que racionalizar el sistema ya desarrollado, implica también la formulación y ejecución de aquellos procesos que permitirán a la organización continuar desarrollándose e innovar.

La segunda premisa es que existen cuatro orientaciones estratégicas dentro de cada industria. El descubrimiento de estos patrones de comportamiento se realizó basándose en estudios de campo en cuatro industrias diferentes -electrónica, proceso de alimentos, salud y libros de texto-, identificando tres tipos recurrentes viables y un cuarto que no representaba un comportamiento estratégico coherente (González, 2001).

Las diferencias principales entre estos tipos se encuentran en la proporción de cambio en el dominio organizativo (Cuadro 2). De acuerdo con el modelo de adaptación organizativa, cada organización escogerá su propio mercado objetivo y desarrollará sus propios productos y/o servicios. Estas decisiones de dominio serán apoyadas por las decisiones apropiadas referentes a la tecnología, la estructura y los procesos de organización (Fraguas, 2003). Así, establecieron los siguientes patrones: defensores, analizadores, prospectores y reactores. Según Miles y Snow (1978) cuando las organizaciones dan respuesta al ciclo adaptativo en cada una de sus dimensiones (empresarial, técnica y administrativa) lo suelen hacer a través de uno de los siguientes patrones estratégicos:

1. Los prospectores están casi siempre atentos al surgimiento de oportunidades de mercado y experimentan regularmente con respuestas para las tendencias emergentes del entorno. Son, con frecuencia, propiciadores de cambios ante los cuales los competidores deben responder y están muy involucrados en innovaciones de productos y mercados, con lo cual, su eficiencia interna puede verse afectada. Las organizaciones suelen ser descentralizadas con estructuras divisionales.

2. Los defensores siguen una estrategia de concentración o enfoque en la 


\section{Características de los tipos estratégicos de Miles y Snow (1978)}

\begin{tabular}{cl}
\hline Tipologías & \multicolumn{1}{c}{ Características } \\
\hline Defensores & $\begin{array}{l}\text { Estrategia de concentración o enfoque en la definición de produc- } \\
\text { to- mercado. Están orientados internamente y enfatizan la reduc- } \\
\text { ción de costes. Sus esfuerzos y atención se dirigen a obtener in- } \\
\text { crementos de eficiencia en sus operaciones. }\end{array}$ \\
& $\begin{array}{l}\text { Están casi siempre atentos a la aparición de oportunidades de } \\
\text { mercado. Regularmente experimentan con respuestas para las } \\
\text { tendencias emergentes del entorno. Están muy involucrados en } \\
\text { innovaciones de productos y mercados. } \\
\text { Híbrido de defensores y prospectores. En entornos estables ope- } \\
\text { ran de forma rutinaria, formal y eficiente. En entornos turbulentos } \\
\text { son seguidores de los competidores más innovadores, adoptan- } \\
\text { do las ideas más prometedoras. } \\
\text { Reactores }\end{array} \quad \begin{array}{l}\text { No responden de forma efectiva a los cambios del entorno. Incon- } \\
\text { sistencia en la adaptación de sus estructuras a las estrategias } \\
\text { cuyo ajuste es normalmente forzado por la presión del entorno. }\end{array}$ \\
\hline Fuente: Miles y Snow (1978). &
\end{tabular}

Fuente: Miles y Snow (1978).

definición de la combinación producto mercado. Como resultado de su estrategia de concentración, no son frecuentes las necesidades de grandes ajustes en su tecnología, estructura o procesos. La atención de los directivos se dirige a obtener incrementos en la eficiencia de sus operaciones actuales. Estos directivos están altamente especializados en su área de operaciones y raramente buscan nuevas oportunidades. La organización tiene una estructura funcional y centralizada.

3. Los analizadores son un híbrido entre los dos tipos anteriores, operan en dos tipos de dominio producto-mercado (el estable y el cambiante) así mostrarán uno u otro comportamiento dependiendo del entorno al que se enfrenten. En entornos estables operan forma rutinaria y eficiente y en entornos turbulentos son seguidores de los competidores más inno- vadores. Analizan y estudian muy bien el riesgo, organizándose en estructuras de tipo matricial.

4. Los reactivos en las empresas de este tipo los directivos perciben frecuentemente el cambio y la incertidumbre en su entorno, pero no responden efectivamente caracterizándose por la ausencia de estrategia. Normalmente la adaptación de sus estructuras a las estrategias es inconsistente y están forzados normalmente por la presión de su entorno.

Según Hambrick (1983) la característica clave implícita en esta tipología es la velocidad mediante la cual una organización cambia sus productos o mercados.

La tercera premisa de esta tipología se refiere al resultado obtenido con cada estrategia, de este modo, Miles y Snow (1978) afirman que si los comporta- 
Posicionamiento estratégico en el ámbito de las franquicias de comida rápida Schlesinger Díaz, María Walesska

mientos prospector, defensivo y analizador se implantan en la organización adecuadamente llevará a un resultado efectivo. La correcta implementación de éstas depende de la coherencia entre los tres elementos que constituyen el ciclo adaptativo de la organización. El rendimiento de los reactores es inferior a los tipos anteriores, por no tener un comportamiento estratégico coherente.

Miles y Snow desarrollan en su modelo, por una parte, las relaciones de la empresa con su entorno y, por otro, las relaciones entre estrategia y estructura. $A$ este respecto Fraguas (2003) recoge las ideas de estos autores y plantea como la estructura y los procesos de la organización influyen en el alcance de los mecanismos de exploración disponibles para la dirección, él resume estas tres premisas de la siguiente manera: a) Las organizaciones actúan para crear sus entornos; b) Las elecciones estratégicas de la dirección conforman la estructura y los procesos de la organización y c) la estructura y los procesos limitan la estrategia, las cuales son explicadas en su trabajo.

Con el tiempo esta limitada actividad de búsqueda, tiende a convertirse en una rutina, de manera que la organización es capaz de hacer ciertas cosas bien y carecer, al mismo tiempo, de capacidades en otras áreas.

Asimismo la tipología de Miles y Snow (1978) permite identificar las empresas que son más proclives a la innovación, MacDaniel y Kolari (1987); constatan la orientación hacia la búsqueda y explotación de nuevos productos/mercados de los prospectores en relación con los defensores, analizadores o reactivos. Los prospectores son pioneros en el desarrollo de innovadores productos y servicios, cambiando constantemente las líneas de productos y aprovechando nuevas oportunidades de mercado (Hambrick, 1980). De estos planteamientos se desprende la primera hipótesis a comprobar:

H1: Las empresas prospectoras innovan más que las empresas analizadoras, reactoras o defensivas.

En segundo lugar en relación a las capacidades de marketing de cada una de las tipologías, los estudios de Morgan et al. (2001) encontraron diferencias significativas entre los prospectores y los defensores, analizadores y reactores, en cuanto a las capacidades de marketing. Morgan et al. (2001) encontraron que los prospectores consistentes en su actitud proactiva dan mayor énfasis en sus capacidades de marketing que los reactores vs. su actitud reactiva, de igual manera los defensores preocupándose más por su ambiente de negocios interno en muchos casos tienen una visión miope en su planificación de marketing, prestando menos atención al mercado, que es realmente donde se encuentran los clientes, proveedores y competidores que los analizadores. De aquí surge entonces la segunda hipótesis a comprobar:

H2: Los analizadores y prospectores dan más énfasis al marketing que los reactores y defensores

De estos planteamientos se pueden concluir que realmente son los prospectores quienes crean el cambio y fuerzan de alguna manera a los defensores y reactores a reaccionar ante estos cambios. Según los resultados de Morgan et al. (2001) referente a las capacidades de marketing no se encontraron diferencias notables entre los analizadores y los 
prospectores, puesto que los analizadores se preocupan mucho por lo que sucede en el ambiente externo (el mercado), lo que los diferencia de ellos en ese aspecto es que son más conservadores a la hora de actuar y de realizar cambios. Los prospectores disfrutan privilegiar la gerencia y dirección comercial, de marketing, y de desarrollo de nuevos productos y servicios, agrandando los productos y servicios que tienen, al tiempo que los defensores más que ir en profundidad con los clientes actuales y con los productos y servicios actuales se orientan a ampliar el "coto de caza" y a ampliar el número de clientes.

\section{Metodología}

El tipo de investigación realizada es de carácter explicativo según Sarabia (1999). Las investigaciones explicativas buscan proporcionar una comprensión del fenómeno que se investiga. Se trata de verificar el fundamento de una hipótesis por medio de un procedimiento metódico diseñado para tal propósito (Sarabia, 1999). Consiste en suma en evaluar el grado de correspondencia real entre el enunciado hipotético y los hechos, teniendo en cuenta que de aprobarse positivamente la hipótesis tan solo significa que en un caso concreto la realidad observable no contradice la hipótesis. En el caso de apreciar alguna discrepancia entre los datos empíricos y la propuesta hipotética, se apela al principio de falsación: se da prioridad a los datos, se rechaza la hipótesis planteada y se procede a sugerir una nueva que se presuma capaz de probar su mayor contenido empírico (Sarabia, 1999).
Según el tipo de diseño de investigación es una investigación No experimental, pues "se realiza sin manipular deliberadamente las variables" (Hernández, Fernández y Baptista, 1998:184). Lo que se hace en una investigación de carácter no experimental es observar fenómenos tal y como se dan en su contexto natural, para después analizarlos (Hernández et al, 1998). Dentro de este tipo de investigación hay una sub-clasificación que serían investigaciones transeccionales y longitudinales. En este caso nos ocupa un diseño no experimental transeccional o transversal según el cual se recolectan datos en un solo momento, en un tiempo único, en esta específicamente se recolectaron en el mes de abril de 2006.

\subsection{Sector Objeto de Estudio}

Para la investigación empírica se seleccionó el sector de comida rápida, considerando que en las últimas décadas el sector de la comida rápida se ha convertido en una industria poderosa (Schlosser, 2004).

El sector de la Comida Rápida ha dejado desde hace tiempo de ser un fenómeno norteamericano y se extiende por todo el planeta a enorme velocidad, sumando un total de más de 88.000 establecimientos repartidos por todo el mundo, según el Boletín emitido por el Observatorio de Corporaciones Transnacionales (2005). Es un sector que avanza sin freno a nivel mundial, la Industria de la Comida Rápida, se encuentra en la actualidad en una etapa de crecimiento sostenido debido fundamentalmente a una fuerte expansión de sus locales ubicados inicialmente en ciudades con gran afluencia de 
Posicionamiento estratégico en el ámbito de las franquicias de comida rápida Schlesinger Díaz, María Walesska

público. España no es ajena a este fenómeno, y se puede observar las diversas compañías que operan en la forma de franquicia y que tienen sus orígenes en países extranjeros.

Se seleccionaron las principales cadenas que operan en España en base a su facturación y participación en el mercado, ellas son: Mc Donald's, Yum Brands (Pizza Hut), Burger King, The Eat Out Group, con Bocatta y Pans \& Company ( $P$ \& $C$ ), Telepizza y Rodilla (Franquicias Hoy, 2006).

En la Tabla 1 se aprecian los datos de facturación y número de establecimientos en España para poder apreciar el posicionamiento y la cuota de mercado que tienen actualmente cada una de estas compañías para el año 2004 y 2005.

El presente estudio se realizó en España, específicamente en la Comunidad Valenciana, conformada por las provincias de Valencia, Castellón y Alicante. Se trabajó específicamente como se detalló en el punto anterior con las principales franquicias de Comida Rápida que operan en esta Comunidad. El estudio se realizó durante los meses de diciembre de 2005 a junio de 2006 .

\subsection{Medición de los conceptos analizados: Estrategias de negocio de Miles y snow (1978)}

El principal problema de la determinación de grupos estratégicos es el de la operacionalización de las estrategias para la posterior identificación de patrones genéricos. Snow y Hambrick (1980) y Zahra y Pearce (1990) recogen los métodos utilizados para tal fin, entre ellos está: inferencia del investigador, autoclasificación de la propia organización a través de la percepción de sus directivos, evaluación externa de expertos y la utilización de indicadores de carácter objetivo. Cada uno de estos métodos tiene puntos fuertes y débiles, éstos últimos pueden ser solventados con la utilización conjunta de varios de ellos.

Mc Daniel y Kolari (1987) utilizan una escala compuesta por cuatro indicadores correspondientes cada una a una tipología diferente, esta escala está ela-

Tabla 1

Datos sobre la franquicia de comida rápida en el Estado Español

\begin{tabular}{lcccc}
\hline Empresa & $\begin{array}{c}\text { FACT 2004 } \\
\text { (miles euros) }\end{array}$ & $\begin{array}{c}\text { FACT 2005 } \\
\text { (miles euros) }\end{array}$ & $\begin{array}{c}\text { Franquicias en } \\
\text { España al 2005 }\end{array}$ & $\begin{array}{c}\text { Franquicias } \\
\text { en la Comunidad } \\
\text { Valenciana }\end{array}$ \\
\hline Mc Donalds & 554.000 & 579.000 & 348 & 46 \\
Telepizza & 320.400 & 318.750 & 530 & 59 \\
Burger King & 240.000 & 268.000 & 394 & 49 \\
Eat Out Group & 200.000 & 210.000 & $311(192$ P\&C & 46 (27 P\&C) \\
Pizza Hut & 78.500 & 70.177 & 136 & 14 \\
Rodilla & 46.540 & 48.230 & 70 & 5 \\
\hline
\end{tabular}

Fuente: Ranking 2006. 
borada a partir de Snow y Hambrick (1980), en estas descripciones se omiten los nombres de cada tipología, y solo se colocan "Tipo 1", "Tipo 2" y así sucesivamente. Este cuestionario fue auto administrado por los directivos de las empresas pertenecientes al estudio, en el cual cada gerente valoraba su empresa, para así poder identificar cuantas empresas pertenecían a cada tipología, de acuerdo a sus percepciones.

Por su parte Cabello, Ruiz, García y Jiménez (2000) analizan el modelo de validación de Miles y Snow propuesto por Dess y Davis (1984) diseñado inicialmente para explicar la tipología de estrategias genéricas de Porter (1980) y que considera 21 factores competitivos explicativos y caracterizadores de dichas estrategias, y lo aplica a la tipología de Miles y Snow, concluyendo que las empresas que se autodefinen en cualquiera de las tipologías de Miles y Snow coinciden en los factores que son mas importantes para ellas en el desarrollo de la misma.

Aragón (1996) considera que los estudios hasta la fecha prestan escasa atención a la validez y a la fiabilidad, creyó oportuno elaborar una herramienta validada para la medición de la estrategia basada en la tipología de Miles y Snow (1978), construida sobre un método multi-ítem, en escala de intervalos y susceptible de ser respondida preferiblemente por un directivo, de manera que se puedan obtener indicadores cuantitativos, validos para su clasificación y comparación, más completos que un cuestionario con una sola pregunta. En concreto se ha aplicado la escala propuesta por Aragón (1996) ya que es considera la más completa y a través de ella se puedan obtener indicadores cuantitativos, validos para su clasificación y comparación, más completos que un cuestionario con una sola pregunta, como es el caso de los utilizados por Snow y Hambrick (1980), además de que esta escala ha sido validada.

Esta escala presentada por Aragón (1996) consta de 18 indicadores que recogen los rasgos en base a las tres dimensiones propuestas: empresarial, tecnológica y administrativa. De esta forma los 6 primeros se refieren a la dimensión empresarial del negocio, los 4 siguientes a la dimensión tecnológica y los 8 últimos se relacionan con la administrativa.

Se ha utilizado una escala tipo Likert del 1 al 5 en la cual cada gerente valoró su empresa según que sus características fueran más cercanas a uno de los polos extremos descritos a ambos lados de la escala. Este proceder recoge la propuesta de Miles y Snow (1978) sobre que las empresas defensivas y atacantes puntuarían en diferentes extremos de una escala, las analizadoras en ciertos temas como defensivas y en otros como atacantes y las reactivas que no seguirían ningún patrón consistente. El cuestionario fue construido de manera que los valores más bajos correspondieran a los rasgos de la estrategia defensiva y los más altos de la atacante.

Para medir la innovación se utilizó la escala propuesta por Henard y Szymanski (2001) y para medir las capacidades del marketing se tomó como referencia la escala elaborada por Conant et al. (1990). Esta escala fue desarrollada cuidadosamente enfocándose en el nivel funcional de las competencias de marketing y es una versión mejorada de la escala utilizada por Snow y Hambrick (1980). 
Posicionamiento estratégico en el ámbito de las franquicias de comida rápida Schlesinger Díaz, María Walesska

\subsection{Población y Muestra}

La investigación se llevó a cabo en España, específicamente en la Comunidad Valenciana. La Comunidad Valenciana es una comunidad autónoma de España conformada por las provincias de Valencia, Castellón y Alicante. La población estuvo compuesta por los gerentes generales (de tienda) de cada franquicia presentes en la Comunidad Valenciana. De acuerdo a la presencia de cada franquicia en la Comunidad Valenciana obtenida directamente de la página web española de cada franquicia para marzo 2006. Se seleccionaron a través del método muestreo sistemático, diferentes franquicias en toda la Comunidad y se repartieron un total de 50 cuestionarios (representan un 23\% de la población), de los repartidos fueron contestados 37 por los gerentes generales de cada franquicia (que representan un $17 \%$ de la población), correspondiendo a Valencia un 78\%, 15\% Alicante y $7 \%$ Castellón, lo cual concuerda con la densidad de franquicias en cada provincia.

Según Chevalier (2004) el muestreo sistemático es cuando se le asigna un número a cada grupo (en este caso cada franquicia) y después se escoge un grupo de cada cinco, diez, etc. Recomienda si la población total es entre 200 y 500 (en nuestro caso es 218) utilizar un $10 \%$. Por lo tanto de acuerdo a lo expuesto la muestra obtenida representa un $17 \%$ de la población, lo cual supera la recomendación de Chevalier (2004) y por consiguiente se considera bastante representativa de la realidad.

\section{Análisis de los datos}

A pesar que los instrumentos fueron validados en cada estudio correspondiente, se quiso verificar los resultados. De acuerdo con las propuestas de Churchill (1979), la revisión de la fiabilidad se analiza a través del coeficiente alpha de Cronbach, el cual analiza la consistencia interna de los ítems de cada una de las dimensiones propuestas.

En relación a la escala de Aragón, el valor de alpha para los seis primeros ítems (dimensión empresarial) alcanza un valor de 0,87 , para los cuatro ítems siguientes (dimensión tecnológica) 0,53 y para los últimos ítems (dimensión administrativa) el coeficiente es igual a 0,56. La escala que mide la innovación el alpha alcanzó un valor de 0,52 y la que mide las capacidades del marketing un valor 0,68.

Si se acepta la recomendación de un valor mínimo de 0,50 según Bagozzi y Yi (1988) para el coeficiente alpha, estarían por encima de lo aceptado.

En primer lugar para caracterizar cada uno de estos grupos dentro de las cuatro clasificaciones que Miles y Snow proponen en su tipología: prospectoras, analizadoras, defensivas y reactivas y de cara al análisis se tomaron los cuestionarios recibidos y se procedió a analizar con el programa SPSS para Windows versión 13.0 (1989-2004), a través de la técnica ANOVA de un factor las medias de cada uno de los 7 tipos de franquicias para cada una de las tres partes del cuestionario que se refería al área empresarial, tecnológica y administrativa. Tomando en cuenta como se mencionó en el marco teórico, que los valores más bajos corres- 
pondieran a los rasgos de la estrategia defensiva y los más altos a los de la prospectora y los términos medio a la analizadora, se pudo obtener que del total, un $32 \%$ de las empresas de comida rápida fueron clasificadas según sus directivos como prospectoras, un $25 \%$ como defensivas y un $41 \%$ como analizadoras, en el grupo de reactivas no salió ninguna empresa y un $2 \%$ no obtuvo ninguna clasificación pudiéndose decir que son outlier (ver Tabla 2). Es importante señalar que aquellas clasificadas como prospectoras pertenecen en su mayoría al sector de Pizzas (Pizza Hut, Telepizza) junto a Mc Donald's.

Ahora bien una vez caracterizadas las empresas se procederá a la contrastación de las hipótesis que se plantearon con anterioridad, pues acorde con Miles y Snow cada tipología muestra una estrategia organizacional diferente, en este caso se analizará el aspecto de la innovación y de las capacidades del marketing.

Para comprobar la primera hipótesis utilizando el programa SPSS se utilizó en primer término la técnica de análisis de la varianza (ANOVA) con los ítems del 19

\section{Tabla 2}

Clasificación según tipología de $M$ \& S del Sector de Comida Rápida en la Comunidad Valenciana

\begin{tabular}{lcc}
\hline Tipología & N & $\%$ \\
\hline Prospectora & 12 & 32 \\
Defensiva & 9 & 25 \\
Analizadora & 15 & 41 \\
Sin Clasificación & 1 & 2 \\
Total & $\mathbf{3 7}$ & $\mathbf{1 0 0}$ \\
\hline
\end{tabular}

Fuente: Elaboración propia. al 25 que median esta variable. El valor $p$ arrojado fue de 0,08 por lo tanto es mayor a 0,05 de manera tal que no se observan diferencias significativas entre al menos una de las medias de los grupos, es decir, que según los resultados las empresas de comida rápida de acuerdo a la percepción de sus directivos innovan por igual a pesar de pertenecer a tipologías diferentes, con lo cual se rechaza la primera hipótesis.

Para comprobar la segunda hipótesis utilizando el programa SPSS se aplicó el mismo procedimiento anterior pero con las medias de los ítems del 33 al 38 correspondientes a medir las capacidades del marketing y en este caso el valor $p$ es 0,003 por lo tanto menor a 0,05 de manera que en este caso si se observan diferencias significativas entre al menos una de las medias de los grupos, posteriormente se aplicó el test de Tukey (Tukey's HSD) el cual arrojó diferencias significativas entre los defensores y los analizadores, y entre los defensores y prospectores, mientras que los analizadores y prospectores no tienen diferencias significativas entre sus medias.

\section{Discusión y Conclusiones}

Tomando en cuenta los resultados obtenidos en primer lugar es importante destacar el hecho que la inclusión de una empresa en alguna de las categorías de la tipología, normalmente se ha basado en el subjetivismo del investigador o en la imprecisión de una escala con pocas facilidades para recoger adecuadamente las particularidades de una empresa determinada. 
Posicionamiento estratégico en el ámbito de las franquicias de comida rápida Schlesinger Díaz, María Walesska

La mayor parte de las veces se venían usando escalas nominales con un solo ítem, las cuales, aparte de su imprecisión, no suelen permitir otra cosa que el uso del tipo de estrategia de una empresa como variable de control en el estudio de relaciones con otras variables. El porcentaje de éxito en la clasificación mediante la herramienta utilizada (Aragón, 1996) supera los alcanzados mediante otras escalas antes utilizadas.

A pesar de esto, sería necesaria la aplicación de esta escala con mayor número de analistas por empresas y en otros sectores. En este caso se aplicó en el Sector de la Comida Rápida.

Según los resultados obtenidos se destaca el hecho que casi la mitad de las empresas (41\%) corresponden a la categoría de analizadoras que según los conceptos manejados son híbridos entre defensores y prospectores, operan en forma rutinaria y formal y son seguidores de los competidores más innovadores, se puede notar que las empresas categorizadas como analizadoras coinciden con aquellas franquicias que ocupan el puesto 3 y 4 en cuanto a volumen de ventas, ellas son Burger King (Internacional), Bocatta y Pans \& Company (nacional).

El $32 \%$ de las empresas coincidieron en la categoría de prospectoras, cuya característica principal es aprovechar al máximo las oportunidades del mercado con nuevos productos y servicios al cliente, dentro de este grupo encontramos a Mc.Donald's, Telepizza y Pizza Hut, la primera (Mc.Donald's) líder en España y a nivel mundial en este sector. Telepizza es un caso específico en este país pues es una franquicia nacional, no internacional como Mc Donald's o Pizza Hut, curio- samente está por encima de Pizza Hut en cuanto a volumen de facturación y sucursales, a pesar de que esta última tiene muchos más años en el país y tiene una gran trayectoria a nivel mundial.

El 25\% fueron categorizadas como defensivas y dentro de esta categoría se encuentra la franquicia Rodilla (nacional), con un menor grado de participación y volumen de facturación que el resto de sus competidores más cercanos, destacando el hecho que en el grupo de reactoras no se clasificó ninguna.

Analizando los resultados que llevaron a rechazar la $\mathrm{H}_{1}$, se observa que no coinciden con los planteamientos del marco teórico en el cual se mencionó el hecho que las empresas prospectoras innovan mas que el resto, se debe hacer notar que el instrumento fue aplicado a los gerentes de las diferentes franquicias y en general la opinión de todos en referencia a esta variable fue muy alta quizá por su grado de pertenencia a la empresa o también dada la numerosa competencia que existe en este sector que en general las franquicias se valen de la innovación constante para poder enfrentarla. Puede ser necesario complementar con otros datos más objetivos que verifiquen estos resultados en futuros estudios.

Por último en el caso de la $\mathrm{H}_{2}$, se comprueba que las empresas catalogadas por los directivos como analizadoras y prospectoras dan más énfasis al marketing que el resto de los grupos, sobretodo en aspectos como promociones, campañas de publicidad y segmentación de mercados en los cuales se obtuvieron las mayores diferencias. Además si analizamos que este grupo de empresas coincide con el hecho de ocupar puestos más 
altos que las empresas catalogadas como defensivas, se podría deducir el hecho que estas empresas presten mas atención y utilicen las técnicas de planificación del marketing las lleva a obtener mejores resultados operativos, aunque este sería otro tema de investigación a comprobar.

Acorde con los planteamientos teóricos presentados es importante que la compañía organice apropiadamente y especifique que estrategias son las más relevantes para el tipo particular al que pertenece y para el cual ha sido la estrategia planificada e implementada. Es importante que los gerentes entiendan su propia realidad, su situación actual, los objetivos y estrategias, y que esto va a variar de la relación constante con su entorno. El modelo propuesto facilita la comprensión de esta situación y la realidad de cada una, así como la realidad de sus competidores.

Los gerentes deben evaluar la consistencia entre la tipología a la cual pertenecen y los elementos de la estrategia empleada, tomando en cuenta que de las tres tipologías encontradas (analizadoras, defensivas y prospectoras) ninguna garantiza el éxito, lo importante es conocer cuál se adapta a su desempeño y ser consistente en ella.

Finalmente esta caracterización refleja la importancia del contexto y de los elementos del sistema, por lo que cualquier nueva concepción sobre estas partes supondrá una modificación en los modelos analizados.

Dentro de las limitaciones de este estudio se destacan las de carácter geográfico puesto que una muestra ma- yor e incluso a nivel de toda España, permitirá en un futuro obtener resultados más generalizables de la población. Asimismo si el instrumento se aplica a nivel de la gerencia corporativa cuyos cargos están más directamente relacionados con la planificación estratégica se pudiera obtener una visión posiblemente diferente de la percibida por los gerentes de tienda.

Sin embargo, como trabajo inicial y original, pues de la bibliografía revisada no se consiguió ningún estudio que aplicara esta tipología al Sector de la Comida Rápida, se obtuvieron interesantes resultados que pueden servir de base para futuras investigaciones, para corroborar los resultados obtenidos o bien sea para aplicarlo en otros países en los cuales se encuentren operando las mismas franquicias y comparar así si la actuación de las mismas en otros países es similar que en España.

Otro tema interesante a tratar es analizar si el país de origen de la franquicia tiene relación con la tipología en la que se encuentra, y poder determinar si las franquicias internacionales y nacionales generalmente corresponden a una categoría específica o por el contrario el país de origen no tiene nada que ver en esto.

De todos modos, a pesar de las limitaciones presentadas se espera que esta investigación colabore en la validación de esta tipología y de pie a futuros estudios en esta área que permita obtener información que ayude a la toma de decisiones de los gerentes en este sector y colabore en el proceso de planificación estratégica. 
Posicionamiento estratégico en el ámbito de las franquicias de comida rápida Schlesinger Díaz, María Walesska

\section{Referencias Bibliográficas}

Abell, D. (1980). Defining the business: Starting point of strategic planning Ed. Prentice-Hall Englewood Cliffs, N.J.

Andrews, K. (1977). The Concept of Corporate Strategy, Dow Jones-Irwin, Homewood, Illinois.

Aragón, J. (1996). La medición de la estrategia empresarial: propuesta y validación de una escala multi-ítem para la medida de la estrategia de negocio según la tipología de Miles y Snow (1978)" Cuadernos de Ciencias Económicas y Empresariales, vol. 31, pgs, 45-65.

Argyris. C. (1985). "Strategy change and defensive routines". Pitman Publishing, Massachusetts.

Cabello, C.; Ruiz, J.; García, M. y Jiménez, A. (2000). "Tipología estratégica de Miles y Snow y factores competitivos" Cuadernos de Economía y Dirección de la empresa. No.7 365-82.

Chevalier, Jacques (2004). elementos adaptados del Socioeconomic and Gender Analysis (SEAGA), Universidad de Carleton, Cánada, revisado el 10/03/06 disponible en www.saspm.com/pdfs /Stakeholder_sampling sp.pdf

Churchill, G.A. (1979). "A paradigm for developing better measures of marketing construcs", Journal of Marketing Research, 16 (1), 64-73.

Bagozzi, R.P. y Yi, Y. (1988). "On the evaluation of structural equation models". Journal of the Academy of Marketing Science, 16(1), 74-94.

Beal, R. (2000). "Competing effectively: Environmental Scanning, Competitive Strategy, and Organizational Performance in Small Manufacturing Firms" Journal of small Business Management, Vol. $38 \mathrm{n}^{\mathrm{a}} 1$ pp. 27-47.
Churchill, Gilbert (1979). "A paradigm for developing better measures of marketing constructs", Journal of Marketing Research.

Chrisman, J.; Hofer, Ch.; Boulton, W. (1988). "Toward a system for classifying business strategies". Academy of Management Review, vol. 13, no 3 , pp. 413-428.

Conant, J.S.; Mokwa, P. y Varadarajan, P. (1990). "Strategic types, distinctive marketing competencies and organizational performance: A múltiple measures-based study". Strategic Management Journal, vol. 11, pp. 365-383.

Dess, G. y Davis, P. (1984). “¿Porter's generis strategies as determinants of strategic group membership an organizational performance", Academy of Management Journal, vol 7, pg 467-88.

Fraguas, R. (2003). "Patologías de los Consejos de Administración en España". IESE Business School, Tesis Doctoral Universidad de Navarra. 295 pags.

Franquicias Hoy (2006). Guía online 2006, disponible en http://www.tormo.com/ monograficos/ranking2006/, revisado el 03.03.06.

Galbrait, C. y Schendel, D. (1983). "An empirical analysis of strategy types". Strategic Management Journal, vol. 4, pp. 153-173.

García y Pérez (2001). "Más allá de la estrategia empresarial: El pensamiento estratégico coherente", Revista Economía Industrial, No.338, 133-146.

García, M. y Sabater, R. (2003). "Relaciones entre estrategia y ciclo de vida de la empresa", Revista Madridmasd, No. 20, España.

González, A. (2001). "Perfil Competitivo y Tecnológico de las Empresas del Sector de la Electrónica. Un Análisis Basado en la Tipología de Estrategias Genéricas de Miles y Snow (1978)" Universidad de Albacete trabajo publicado en 
http://www.uclm.es/AB/fcee/D trabajos/5-2001-1.PDF.

Hambrick, D. (1980). "Operationalizing the concept of business-level strategy in research" The Academy of Management Review, pp 567-575.

Hambrick, D. (1982). "Environmental scanning and organization strategy". Strategic Management Journal, vol 3, pp. 159-174

Hambrick, D. (1983). "Some tests of the effectiveness of functional attributes of Miles and Snow's strategic types", Academy of Management Journal, Vol 26 No. 1, pp 5-26.

Henard, D. y Szymanski, D. (2001). "Why some new products are more successful than others", Journal of Marketing Research, XXXVIII, August, 362-375.

Hernández, Roberto; Fernández, Carlos y Baptista, Pilar (1998). Metodología de la Investigación, Mc Graw Hill, 2da. Edición, México.

Manu, F. y Sriram (1996). "Innovation, marketing strategy, environment and perfomance", Journal of Business Research, Vol 35, pp 79-91.

Mc Alexander, J.; Becker, B. y Kaldenberg, D. (1993). "Positioning Health Care Services: Yellow Pages Advertising and Dental Practice Performance", Journal of Health Care Marketing, № 13, winter, 54-57.

Mc Daniel S. y Kolari, J. (1987) "Marketing Strategy Implications of the Miles and Snow Strategic Tipology" Journal of Marketing, vol 51, pg. 19-30.

Miles R. y Snow C. (1978). "Organizacional Strategy, Structure and Process", New York: Mc.Graw Hill.

Mintzberg, H. (1978). "Patterns of strategy formation”, Management Science, 24

Mintzberg, H. (1988). "Generic Strategies: Toward a comprehensive framework".
Advances in Strategic Management, vol. 5, pp.1-67.

Morgan R., Strong C. y Mc Guiness T. (2001). "Prospector Strategy \& Competitive Attributes - A Multiattribute Analysis Of Strategy Types" School of Management Business Research Papers, Wales, UK.

Nut, P. y Bachoff, R. (1992). "Strategic management of public and third sector organizations". San Francisco: Josey Bass Publishers.

Pearce, J. y Zahra, S. (1990). "Resarch evidence on the Miles-Snow Typology", Journal of Management, Dec 1990 pg. 751- 768

Porter, M. (1985). Competitive Strategy, Free Press, New York.

Ramsay, B. (1983). "Brand efficiency: lessons from the USA", ADMAP, Vol. 19 No.11, pp.556-63.

Ries, A. y Trout, J. (1981). Positioning, The battle for your mind, Warner Books McGraw-Hill Inc., New York, 1981.

Ries, A. y Jack Trout (2000). 'Focus in a fuzzy world" Executive Excellence. Apr 2000. Vol. 17, Iss. 4.

Ramsay, B. (1983). Brand Efficiency: Lessons from the USA. ADMAP, Vol. 19, №11, 556-63.

Sarabia, F. (1999). Metodología para la investigación en marketing y dirección de empresas. Ediciones Pirámide, Madrid, España.

Schlosser, E. (2004). Slow Food Nation, en The Ecologist, Disponible en http://www.theecologist.org/archive article.html?article $=454 \&$ category= 53, consultado el 29.12.05.

Segev, E. (1989). "A systematic comparative analysis of two business-level strategic typologies", Strategic Management Journal, Vol.10, pp 487-505.

Shortell, S. y Zajac, E. (1990). "Perceptual and archival measures of Miles and 
Posicionamiento estratégico en el ámbito de las franquicias de comida rápida Schlesinger Díaz, María Walesska

Snow's strategic types: a comprehensive assessment of reliability and validity", Academy of Management Journal, Vol.33 No. 4, pp 817-32.

Snow, C. y Hambrick, C. (1980). "Measuring Organizational Strategies: Some Theorical and Methodological Problems", Academy of Management Review, 5 (4), 527-38.

SPSS (1989-2004). SPSS for Windows (Version 12.0): SPSS, Inc.
Trout, J. (1969). "Positioning" is a game people play in today's me-too market place", Industrial Marketing, Vol.54, No.6, (June 1969), pp.51-55.

Walker, R. y Ruekert, O. (1987). "Marketing's Interaction with Other Functional Units: A Conceptual Framework and Empirical Evidence, Journal of Marketing, Vol. 51, No. 1 (Jan., 1987), pp. 1-19.

\section{ESCALA}

1. El campo de actuación en el que la empresa desarrolla actualmente su actividad es:

\begin{tabular}{|l|l|l|}
\hline $\begin{array}{l}\text { Estrecho (áreas relacionadas) y con } \\
\text { pocas perspectivas de ser ampliado }\end{array}$ & 12345 & $\begin{array}{l}\text { Amplio (diversificación en áreas) y } \\
\text { en continuo desarrollo }\end{array}$ \\
\hline
\end{tabular}

2. Debido a la preocupación por la evolución del entorno lo que solemos hacer es:

\begin{tabular}{|l|l|l|}
\hline $\begin{array}{l}\text { Estudiar en profundidad la evolución } \\
\text { del entorno dentro de su actual campo } \\
\text { de actuación }\end{array}$ & 12345 & $\begin{array}{l}\text { Estudiar lo mejor que es posible las } \\
\text { condiciones y acontecimientos del } \\
\text { entorno en general }\end{array}$ \\
\hline
\end{tabular}

3. La mejor manera de enfrentarse a la competencia en este sector es:

\begin{tabular}{|c|c|c|c|c|c|c|}
\hline $\begin{array}{l}\text { Una continua evolución de la gama de } \\
\text { producto que los hagan atractivos al } \\
\text { público (innovador, diferente, etc.) }\end{array}$ & & 2 & 3 & 4 & 5 & $\begin{array}{l}\text { Una fuerte resistencia en defensa } \\
\text { de sus productos actuales, mejoras } \\
\text { especialmente en precios y/o en } \\
\text { servicio }\end{array}$ \\
\hline
\end{tabular}

4. La manera en que esta empresa plantea su crecimiento es:

\begin{tabular}{|l|llllll|l|}
\hline $\begin{array}{l}\text { A través de la penetración en merca- } \\
\text { dos (ganando participación de mer- }\end{array}$ & $\begin{array}{llllll}1 & 2 & 3 & 4 & 5 & \begin{array}{l}\text { A través del desarrollo de nuevos } \\
\text { productos y mercados }\end{array} \\
\text { cado) }\end{array}$
\end{tabular}

5. La opinión de esta empresa ante oportunidades en el entorno podría ser:

\begin{tabular}{|c|c|c|c|c|c|c|}
\hline $\begin{array}{l}\text { Hay que aprovechar rápidamente las } \\
\text { posibles oportunidades }\end{array}$ & & 2 & 3 & 4 & 5 & $\begin{array}{l}\text { Hay que analizarlas y estudiar con } \\
\text { cautela las posibles oportunidades }\end{array}$ \\
\hline
\end{tabular}


6. Cuando aparecen nuevos productos en el seno de la empresa se encuentran:

\begin{tabular}{|c|c|c|c|c|c|}
\hline $\begin{array}{l}\text { En áreas emergentes, dondequiera } \\
\text { que hava existido la posibilidad }\end{array}$ & & 12 & 2 & & 4 \\
\hline
\end{tabular}

7. Por lo que respecta al proceso tecnológico de la empresa, la principal preocupación es:

\begin{tabular}{|l|lllll|l|}
\hline $\begin{array}{l}\text { Contar con tecnologías eficientes en } \\
\text { costes }\end{array}$ & 1 & 2 & 3 & 4 & 5 & $\begin{array}{l}\text { Contar con tecnologías flexibles e } \\
\text { innovadoras }\end{array}$ \\
\hline
\end{tabular}

8. La tecnología con la que actualmente cuenta la empresa:

\begin{tabular}{|l|lllll|l|}
\hline Es homogénea, basada en un núcleo & 1 & 2 & 3 & 4 & 5 & Es una tecnología múltiple, no ba- \\
\hline
\end{tabular}
tecnológico desarrollado sada en ningún núcleo tecnológico

\section{El grado de rutina impuesto por el proceso tecnológico es:}

\begin{tabular}{|c|c|c|c|c|c|}
\hline $\begin{array}{l}\text { ción no es completa } \\
\text { nte marca el ritmo y }\end{array}$ & 12 & 3 & & & $\begin{array}{l}\text { Alto, el proceso tecnológico marca } \\
\text { el ritmo y la forma del trabajo reali- } \\
\text { zado }\end{array}$ \\
\hline
\end{tabular}

10. La tecnología con la que actualmente contamos es:

\begin{tabular}{|c|c|c|c|c|c|}
\hline $\begin{array}{l}\text { Una de las más avanzadas del merca- } \\
\text { do, ya que el esfuerzo en su mejora } \\
\text { continua, ha reducido sus costes a ni- } \\
\text { veles muy competitivos }\end{array}$ & 12 & 3 & 4 & & $\begin{array}{l}\text { Una de las más avanzadas de } \\
\text { mercado, ya que si bien sus costes } \\
\text { nos son bajos, nos permite hace } \\
\text { trabajos únicos en el mercado }\end{array}$ \\
\hline
\end{tabular}

\section{Las personas más influyentes en la organización se encuentran:}

\begin{tabular}{|c|c|c|c|c|}
\hline $\begin{array}{l}\text { Entre los expertos y jefes de las áreas } \\
\text { de producción y finanzas }\end{array}$ & 12 & 3 & & $\begin{array}{l}\text { Entre los expertos y jefes de las } \\
\text { áreas de marketing e Investigación } \\
\text { y Desarrollo }\end{array}$ \\
\hline
\end{tabular}

12. Las personas que ocupan actualmente los puestos más altos de la empresa:

\begin{tabular}{|c|c|c|c|c|c|}
\hline $\begin{array}{l}\text { Proceden de ascensos dentro de la } \\
\text { misma empresa }\end{array}$ & $\begin{array}{lll}12 & 2\end{array}$ & 3 & 4 & & $\begin{array}{l}\text { Proceden de personal contratado } \\
\text { fuera de la empresa en momentos } \\
\text { necesarios }\end{array}$ \\
\hline
\end{tabular}


Posicionamiento estratégico en el ámbito de las franquicias de comida rápida Schlesinger Díaz, María Walesska

\section{La planificación en esta empresa es:}

\begin{tabular}{|l|lllll|l|}
\hline $\begin{array}{l}\text { Tremendamente abierta, imposible } \\
\text { de terminar antes de la acción }\end{array}$ & 1 & 2 & 3 & 4 & 5 & $\begin{array}{l}\text { Tremendamente rigurosa y estruc- } \\
\text { turada }\end{array}$ \\
\hline
\end{tabular}

14. El tipo de estructura con el que cuenta la organizaciones:

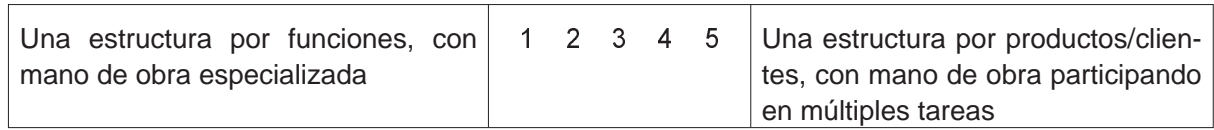

15. En relación a la manera de ejercer el control en la empresa nos encontramos con:

\begin{tabular}{|c|c|c|c|c|c|}
\hline $\begin{array}{l}\text { Un control descentralizado, apoyado } \\
\text { en sist. de información horizontales } \\
\text { (interdepartamentales) }\end{array}$ & 12 & 23 & 3 & 4 & $\begin{array}{l}\text { Un control centralizado apoyado en } \\
\text { sistemas de información vertical (de } \\
\text { los gerentes a los empleados) }\end{array}$ \\
\hline
\end{tabular}

16. La coordinación entre los diferentes miembros de la empresa cuenta con:

\begin{tabular}{|c|c|c|c|c|c|}
\hline $\begin{array}{l}\text { Mecanismos de coordinación comple- } \\
\text { jos y conflictos resueltos mediante re- } \\
\text { laciones personales }\end{array}$ & 12 & 23 & 4 & 5 & $\begin{array}{l}\text { Mecanismos de coordinación sim- } \\
\text { ples y resolución de conflictos a tra- } \\
\text { vés de canales jerárquicos }\end{array}$ \\
\hline
\end{tabular}

17. Cuando hay que determinar la bondad del resultado empresarial, la empresa prefiere fijarse en:

\begin{tabular}{|l|lllll|l|}
\hline $\begin{array}{l}\text { Resultado medido en comparación a } \\
\text { años anteriores }\end{array}$ & 1 & 2 & 3 & 4 & 5 & $\begin{array}{l}\text { Resultado medido respecto de los } \\
\text { competidores }\end{array}$ \\
\hline
\end{tabular}

18. El grupo de personas dominantes en la empresa:

\begin{tabular}{|c|c|c|c|c|c|}
\hline $\begin{array}{l}\text { Es bastante estable, por lo que la coa- } \\
\text { lición dominante está muy experimen- } \\
\text { tada }\end{array}$ & 12 & & & & $\begin{array}{l}\text { Varía bastante según los aconteci- } \\
\text { mientos, la experiencia de la coali- } \\
\text { ción dominante puede no ser larga }\end{array}$ \\
\hline
\end{tabular}




\section{II.- En materia de innovación, señale mediante una cruz como valoría la posición de su empresa:

$1=$ Muy baja $\quad$ y $\quad 5=$ Muy alta

19. Superioridad (y/o diferenciación) respecto al resto de productos competidores

20. Grado en que el producto se adapta a las necesidades/gustos de los clientes

21. Grado de congruencia entre el precio del producto y el resultado que reporta

21. Grado de congruencia entre el precio del producto y el resultado que reporta

22. Sofisticación tecnológica percibida en el producto

23. Novedosidad/originalidad percibida en el producto

24. Grado en el que la empresa desarrolla nuevos productos para lanzarlos al mercado

25. Grado en el que la empresa acomete actividades de prelanzamiento (generación/búsqueda de ideas, investigación de mercados, análisis financieros...)

\section{En materia de marketing, señale mediante una cruz como valoría la preocupación de su empresa por:

$1=$ Muy baja $\quad$ y $\quad 5=$ Muy alta

26. Ofrecer variedad de productos

27. Ofrecer beneficios variados al cliente

28. Ofrecer alta tecnología

29. Conocer a sus clientes

30. Investigar sobre sus competidores

31. Planificación en Marketing

32. Desarrollar habilidades para segmentar mercados

33. Desarrollar habilidades para diferenciar su servicio de la competencia

34. Calidad de servicio

35. Desarrollar campañas de publicidad

36. Desarrollar promociones (bajar precios, regalos, $2 \times 1$, etc.)

37. Mejorar instalaciones

38. Tomar en cuenta las opiniones de los clientes (positivas o negativas) 